\title{
Alkaloid profiling of Galanthus woronowii Losinsk. by GC-MS and evaluation of its biological activity
}

Buket BOZKURT, Ahmet EMİR, Gülen İrem KAYA, Mustafa Ali ÖNÜR, Strahil BERKOV, Jaume BASTİDA, Nehir ÜNVER SOMER

\begin{abstract}
The alkaloid profiles of the aerial parts and the bulbs of Galanthus woronowii Losinsk. were analyzed by means of Gas Chromatography-Mass Spectrometry (GC-MS). Totally, twentyeight compounds were detected. Galanthamine and galanthine were found to be the main alkaloids both in the extracts of the aerial parts and the bulbs. Most of the identified compounds were lycorine and galanthamine-type Amaryllidaceae alkaloids.
\end{abstract}

In addition, the acetylcholinesterase (AChE) inhibitor potentials of the extracts prepared from the aerial parts and bulbs were determined by in vitro Ellman's method and both of the extracts were found to exhibit significant activity (aerial parts: $\mathrm{IC}_{50}=$ $0.027 \mu \mathrm{g} / \mathrm{mL}$ and bulbs: $\mathrm{IC}_{50}=0.084 \mu \mathrm{g} / \mathrm{mL}$ ).

Keywords: Galanthus woronowii; Amaryllidaceae; GC-MS; alkaloids.
Buket Bozkurt, Ahmet Emir, Gülen İrem Kaya, Mustafa Ali Önür, Nehir Ünver Somer

Department of Pharmacognosy, Faculty of Pharmacy, Ege University, 35100, Izmir, Türkiye

Strahil Berkov

AgroBio Institute, 8 Dragan Tzankov Blvd., 1164-Sofia, Bulgaria

Jaume Bastida

Departament de Productes Naturals, Biologia Vegetal i Edafologia, Facultat de Farmacia, Universitat de Barcelona, Av. Diagonal 643, 08028 Barcelona, Spain

Corresponding Author:

Nehir Ünver Somer

e-mail:nehir.somer@ege.edu.tr; nehirunver@hotmail.com

Submitted / Gönderilme: 01.03.2017 Revised / Düzeltme: 29.06.2017 Accepted / Kabul: 06.07.2017

How to cite this article: Bozkurt B, Emir A, Kaya GI, Önür MA, Berkov S, Bastida J, Ünver Somer N. Alkaloid profiling of Galanthus woronowii Losinsk. by GC-MS and evaluation of its biological activity. Marmara Pharm J 2017; 21 (4): 915-920

\section{Introduction}

The genus Galanthus L. belonging to the family Amaryllidaceae, is represented by 14 taxa and one hybrid in Turkey. Among these taxa, Galanthus woronowii Losinsk., is found in north-eastern part of Turkey [1, 2]. Previous phytochemical investigations have revealed that the genus Galanthus L. (Amaryllidaceae) is a very rich source of chemically diverse alkaloids, a quite number of them reported to be new compounds $[3,4]$.

G. woronowii is also distributed in Caucasus, Transcaucasus, Southern Russia and Georgia. Moreover, G. woronowii with broad green leaves is an attractive plant used in gardening and also for decorative purposes [1, 2]. Previously, G. woronowii has been a subject of several phytochemical studies such as isolation of galanthamine, lycorine, tazettine, galanthine and sanguinine [5-7]. In the present study, the alkaloid profile of G. woronowii was investigated by GC-MS. To the best of our knowledge, this is the first report of a GC-MS study on the alkaloids of $G$. woronowii of Turkish origin. Moreover, AChE inhibitory activity potentials of the extracts prepared from $G$. woronowii were examined spectrophotometrically by using a micro-plate assay modified from in vitro Ellman's method with 96-well micro-plate reader [8]. 


\section{Materials and Methods}

\subsection{Plant Material}

Galanthus woronowii Losinsk. was collected in 2009 from Derepazar1-Rize north-east of Turkey. The plant was identified by Prof. M. Ali Önür (Ege University). A voucher specimen (No: 1417) has been deposited in the Herbarium of the Department of Pharmacognosy, Faculty of Pharmacy, Ege University.

\subsection{Extract Preparation}

The extracts were prepared from the aerial parts and the bulbs of G. woronowii. Air-dried, powdered plant material $(500 \mathrm{mg}$ ) was separately extracted 3 times with methanol $(5 \mathrm{~mL})$ at room temperature. The solvent was evaporated under reduced pressure, the residues dissolved in $10 \mathrm{~mL} 2 \%$ sulfuric acid and the neutral compounds were removed with diethyl ether $(3 \times 10 \mathrm{~mL})$. The acidic aqueous phases were basified with $25 \%$ ammonia to $\mathrm{pH} 9-10$ and the alkaloids were extracted with chloroform $(3 \times 10 \mathrm{~mL})$. The combined chloroform extracts were then dried over anhydrous sodium sulphate, filtered and the organic solvent was distilled in vacuo to afford the alkaloidal extract [9]. Prior to the GC/MS analysis, the extracts were dissolved in $\mathrm{CH}_{3} \mathrm{OH}(5 \mathrm{mg}$ extract in $250 \mu \mathrm{L} \mathrm{CH}_{3} \mathrm{OH}$ ).

\subsection{Gas Chromatography-mass spectrometry (GC/MS)}

The alkaloids were detected by capillary gas chromatographymass spectrometry (GC/MS) on a Hewlett-Packard 6890+MSD 5975, (Hewlett-Packard, Palo Alto, CA, USA) operating in the electron impact mode (EI, $70 \mathrm{eV}$ ). The temperature conditions worked with the following program: $100-180^{\circ} \mathrm{C}$ at $15^{\circ} \mathrm{C} \min ^{-1}, 180-300^{\circ} \mathrm{C}$ at $5^{\circ} \mathrm{C} \min ^{-1}$ and a 10 min hold at $300^{\circ} \mathrm{C}$. Injector temperature was $250^{\circ} \mathrm{C}$. Helium was used as a carrier gas at a flow rate of $0.8 \mathrm{~mL} \mathrm{~min}^{-1}$ The split ratio was 1:20. A HP-5 MS column $(30 \mathrm{~m} \mathrm{x} 0.25 \mathrm{~mm} \times$ $0.25 \mu \mathrm{m}$ ) was used.

The spectra of co-eluting chromatographic peaks were determined by the use of AMDIS 2.64 (NIST, National Institute of Standardization and Technology, Gaithersburg, MD, USA). The alkaloids were identified by comparing the mass spectral fragmentation with standard reference spectra from the NIST 05 database (NIST Mass Spectral Database, PC-Version 5.0 (2005), National Institute of Standardization and Technology, Gaithersburg, MD) or applying cochromatography with previously isolated authentic standards and in comparison with data obtained from the literature. The percentage of the total ion current (TIC) and the Kovats retention indices (RI) for each compound were given in Table 1. The area of the GC-MS peaks depends both on the concentration of the corresponding compound and on the intensity of their mass spectral fragmentation. The data given in Table 1 do not reveal a real quantification. However, they can be used for a relative comparison of the alkaloids.

\subsection{Acetylcholinesterase (AChE) Inhibitory Activity}

AChE inhibitory activity was assessed spectrophotometrically by using a micro-plate assay modified from Ellman's method with 96-well micro-plate reader, as previously described $[8,10]$. The dilutions of each sample were prepared to get final concentrations ranging between $75-7.5 \times 10^{-5} \mu \mathrm{g} / \mathrm{mL}$. The inhibitory concentration of $50 \%\left(\mathrm{IC}_{50}\right)$ was determined by a GraphPad Prism V3.0 software (GraphPad Software, San Diego, CA, USA). Galanthamine was used as a positive control.

\section{Results and Discussion}

Totally, twenty-eight compounds were detected by GC/ MS (Table 1). The structures of the alkaloids were given in Figure 1. The identified alkaloids belonged to galanthamine-, lycorine-, tazettine-, and phenanthridinetype Amaryllidaceae alkaloids plus two other bases tyramine (1) and hordenine (2) were reported. The results of the chromatographic determination indicated that $G$. woronowii is a rich source of lycorine- and galanthamine-type Amaryllidaceae alkaloids. Galanthamine, an anti-Alzheimer drug [11] (4) and galanthine (15) were found as major alkaloids in both of the extracts. Also, lycorine (18) an alkaloid with different biological activities [12], methylleucotamine (23) and narwedine (8) were detected. In addition to these findings, one tazettine-type alkaloid, deoxytazettine (11) and one phenanthridine-type alkaloid, 5,6-dihydrobicolorine (3) were determined. Interestingly, two compounds (GW-1 and $\mathrm{GW}-2)(\mathbf{1 4}, \mathbf{2 1})$ showing mass spectral fragmentation of lycorine-type alkaloids were recorded by GC-MS in the alkaloidal extracts.

Previous karyological research on Turkish Galanthus populations indicated a polymorphism existed among different karyotypes and SAT -chromosomes which might be important in the alkaloidal variation [13-15]. Up to date, phytochemical studies carried on various Galanthus species revealed that they were an intruguing source for alkaloids 
Table 1. Relative composition of the alkaloids in Galanthus woronowii

\begin{tabular}{|c|c|c|c|c|c|c|}
\hline \multicolumn{6}{|c|}{ Content $^{\star}$} & \multirow[b]{2}{*}{ References } \\
\hline Compound & RT & RI & $m / z$ (relative intensity,\%) & $\begin{array}{l}\text { A e r i a l } \\
\text { Parts }\end{array}$ & Bulbs & \\
\hline Tyramine $^{a}(1)$ & 7.21 & 1414 & $\begin{array}{llll}137\left(\mathrm{M}^{+}, 16\right), & 108(100), & 107(60), & 91(6), \\
77(24), 65(3) & & & \end{array}$ & $\mathrm{t}$ & $\mathrm{t}$ & {$[17]$} \\
\hline Hordenine $^{\mathrm{a}}(2)$ & 12.72 & 1467 & $\begin{array}{l}165\left(\mathrm{M}^{+}, 1\right), 121(2), \quad 107(5), 91(3), 77(6), \\
58(100)\end{array}$ & - & $\mathrm{t}$ & {$[18]$} \\
\hline 5,6-Dihydrobicolorine ${ }^{\mathrm{b}}(3)$ & 20.82 & 2321 & $\begin{array}{l}239\left(\mathrm{M}^{+}, 42\right) \quad 238(100), \\
118(5), 90(3)\end{array}$ & $\mathrm{t}$ & $\mathrm{t}$ & [19] \\
\hline Galanthamine ${ }^{c}(4)$ & 21.41 & 2405 & $\begin{array}{l}287\left(\mathrm{M}^{+}, 89\right), 286(100), 270(13), 244(26), \\
230(13), 216(32), 174(26), 128(9), 115(13)\end{array}$ & 36.2 & 36.4 & NIST05, [20], S \\
\hline Sanguinine $^{c}(5)$ & 21.56 & 2413 & $\begin{array}{l}273\left(\mathrm{M}^{+}, 100\right), 272(86), 256(22), 230(20) \\
225(17), 216(46), 212(24), 211(34), 202(37)\end{array}$ & 0.1 & - & {$[17]$} \\
\hline$N$-Demethylgalanthamine ${ }^{c}(\mathbf{6})$ & 21.90 & 2442 & $\begin{array}{l}273\left(\mathrm{M}^{+}, 99\right), 272(100), 230(36), 202(27), \\
174(14)\end{array}$ & 0.3 & 1.2 & {$[21]$} \\
\hline Epigalanthamine $^{c}(7)$ & 22.07 & 2454 & $\begin{array}{l}287\left(\mathrm{M}^{+}, 86\right), \quad 286(100), \quad 244(20), \quad 230(15), \\
216(62), 174(25)\end{array}$ & $\mathrm{t}$ & $\mathrm{t}$ & {$[22]$} \\
\hline Narwedine $^{c}(\mathbf{8})$ & 22.47 & 2482 & $\begin{array}{l}285\left(\mathrm{M}^{+}, 85\right), \quad 284(100), \quad 242(20), \quad 228(11), \\
216(20), 199(18), 174(29)\end{array}$ & 1.6 & 1.1 & {$[17]$} \\
\hline Anhydrolycorine d ${ }^{\text {(9) }}$ & 22.91 & 2502 & $\begin{array}{l}251\left(\mathrm{M}^{+}, 45\right), \quad 250(100), \quad 220(2), \quad 192(11), \\
191(10), 165(3), 124(6)\end{array}$ & 0.4 & 0.1 & NIST05, [21] \\
\hline$O$-acetylgalanthamine ${ }^{c}(10)$ & 23.35 & 2534 & $\begin{array}{l}329\left(\mathrm{M}^{+}, 37\right), 328(31), \quad 270(100), 226(11), \\
216(25), 174(11)\end{array}$ & 0.1 & - & [17], [23] \\
\hline Deoxytazettine $^{\mathrm{e}}(\mathbf{1 1})$ & 23.47 & 2541 & $\begin{array}{l}315\left(\mathrm{M}^{+}, 21\right), \quad 300(16), \quad 250(8), \quad 231(100), \\
211(16), 169(6), 141(9)\end{array}$ & - & 0.1 & {$[21]$} \\
\hline Assoanine $^{d}(\mathbf{1 2})$ & 23.98 & 2579 & $\begin{array}{l}267\left(\mathrm{M}^{+}, 50\right), \quad 266(100), \quad 250(29), \quad 222(8), \\
205(3), 193(7), 180(7)\end{array}$ & 0.1 & 0.1 & {$[11], S$} \\
\hline 11,12-Didehydroanhydrolycorine (13) & 24.42 & 2602 & $\begin{array}{l}249\left(\mathrm{M}^{+}, 59\right), \quad 248(100), \quad 190(26), \quad 163(8), \\
95(12)\end{array}$ & $\mathrm{t}$ & $\mathrm{t}$ & {$[24]$} \\
\hline GW-1 $1^{\mathrm{d}}(\mathbf{1 4})$ & 25.37 & 2668 & $\begin{array}{l}265\left(\mathrm{M}^{+}, 85\right), \quad 264(100), 248(17), \quad 191(13), \\
178(18)\end{array}$ & $\mathrm{t}$ & $\mathrm{t}$ & - \\
\hline Galanthine ${ }^{\mathrm{d}}(\mathbf{1 5})$ & 25.99 & 2709 & 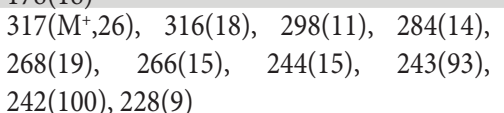 & 50.5 & 56.2 & {$[18]$} \\
\hline $\begin{array}{l}\text { 1-O-acetyl-9-O-methylpseudolycorine }{ }^{\mathrm{d}} \\
\text { (16) }\end{array}$ & 26.36 & 2729 & $\begin{array}{l}345\left(\mathrm{M}^{+}, 34\right), 284(27), \quad 243(77), \quad 242(100), \\
228(9)\end{array}$ & $\mathrm{t}$ & 0.5 & [7] \\
\hline Incartine ${ }^{\mathrm{d}}(\mathbf{1 7})$ & 26.49 & 2739 & $\begin{array}{l}333\left(\mathrm{M}^{+}, 43\right), \quad 332(100), \quad 296(28), \quad 259(71), \\
258(86)\end{array}$ & $\mathrm{t}$ & $\mathrm{t}$ & [17] \\
\hline Lycorine $^{\mathrm{d}}(\mathbf{1 8})$ & 26.59 & 2743 & $\begin{array}{l}287\left(\mathrm{M}^{+}, 30\right), \quad 268(24), \quad 250(32), \quad 227(68), \\
226(100), 211(5), 147(9)\end{array}$ & 4.3 & 3.5 & [21] \\
\hline 9-O-methylpseudolycorine ${ }^{\mathrm{d}}(\mathbf{1 9})$ & 26.87 & 2788 & $303\left(\mathrm{M}^{+}, 35\right), 284(24), 243(86), 242(100)$ & 0.1 & 0.5 & {$[25]$} \\
\hline Sternbergine ${ }^{\mathrm{d}}(\mathbf{2 0})$ & 27.09 & 2801 & $\begin{array}{l}331\left(\mathrm{M}^{+}, 36\right), \quad 270(27), \quad 254(10), \quad 252(14), \\
229(67), 228(100), 43(9)\end{array}$ & - & 0.1 & [25], S \\
\hline $\mathrm{GW}-2{ }^{\mathrm{d}}(\mathbf{2 1})$ & 27.52 & 2841 & $\begin{array}{l}297\left(\mathrm{M}^{+}, 62\right), \quad 296(100), \quad 280(17), \quad 266(4), \\
252(5), 224(3), 149(6)\end{array}$ & 2.7 & - & - \\
\hline 2-O-acetyllycorine ${ }^{\mathrm{d}}(\mathbf{2 2})$ & 27.78 & 2879 & $\begin{array}{l}329\left(\mathrm{M}^{+}, 18\right), 268(76), 250(100), 240(12), \\
226(23), 192(11)\end{array}$ & 0.2 & - & {$[26,27]$} \\
\hline Methylleucotamine ${ }^{c}(23)$ & 28.45 & 2956 & $\begin{array}{l}373\left(\mathrm{M}^{+}, 100\right), 330(2), 270(100), 216(18), \\
165(9)\end{array}$ & 1.5 & 0.1 & {$[28]$} \\
\hline $\begin{array}{l}\text { 3-O-(3'-acetoxybutanoyl) } \\
\text { galanthamine }(24)\end{array}$ & 29.92 & 3070 & $\begin{array}{l}415\left(\mathrm{M}^{+}, 19\right), \quad 384(9), \quad 286(2), \quad 270(100), \\
216(18)\end{array}$ & 0.2 & - & [29] \\
\hline 2-O-2'-butenoyllycorine ${ }^{\mathrm{d}} \mathbf{( 2 5 )}$ & 30.95 & 3125 & $\begin{array}{l}355\left(\mathrm{M}^{+}, 21\right), \quad 269(66), 268(83), \quad 250(100), \\
226(35), 192(6), 147(11)\end{array}$ & $\mathrm{t}$ & - & {$[18,30]$} \\
\hline 2-O-2'-butenoyllycorine isomer ${ }^{\mathrm{d}}(\mathbf{2 6})$ & 31.54 & 3156 & $\begin{array}{l}355\left(\mathrm{M}^{+}, 21\right), \quad 269(64), \quad 268(88), \quad 250(100), \\
226(31), 192(10), 147(9)\end{array}$ & 0.4 & $\mathrm{t}$ & {$[18,30]$} \\
\hline $\begin{array}{l}\text { 2-O-(3'-hydroxybutanoyl)lycorine }{ }^{d} \\
\text { (27) }\end{array}$ & 32.83 & 3226 & $\begin{array}{l}373\left(\mathrm{M}^{+}, 13\right), \quad 372(9), \quad 269(51), \quad 268(70) \\
251(38), 250(100), 226(32), 147(7)\end{array}$ & 0.9 & 0.1 & [29] \\
\hline $\begin{array}{l}2-O \text {-(3'-Acetoxybutanoyl)lycorine }{ }^{d} \\
(28)\end{array}$ & 34.05 & 3336 & $\begin{array}{l}415\left(\mathrm{M}^{+}, 13\right), \quad 414(6), \quad 269(57), \quad 268(70), \\
250(100), 227(19), 226(28), 192(11)\end{array}$ & 0.5 & - & {$[18]$} \\
\hline
\end{tabular}

a:other type b: phenanthridine-type c: galanthamine-type d: lycorine-type e: tazettine-type S: Standard t:trace $<0.1$

${ }^{*}$ The area of the GC/MS peaks depends not only on the concentration of the related compounds, but also on the intensity of their mass spectral fragmentation. 
<smiles>[R]N([R1])CCc1ccc(O)cc1</smiles>

$1 \mathrm{R}_{1}=\mathrm{R}_{2}=\mathrm{H}$

$2 \mathrm{R}_{1}=\mathrm{R}_{2}=\mathrm{CH}_{3}$<smiles>CNCc1cc2c(cc1-c1ccccc1C)OCO2</smiles>

3<smiles>O=C(O)c1cc2c(cc1O)CN1CCc3cccc-2c31</smiles>

$9 \mathrm{R}_{1}+\mathrm{R}_{2}=\mathrm{CH}_{2}$

$12 \mathrm{R}_{1}=\mathrm{R}_{2}=\mathrm{CH}_{3}$

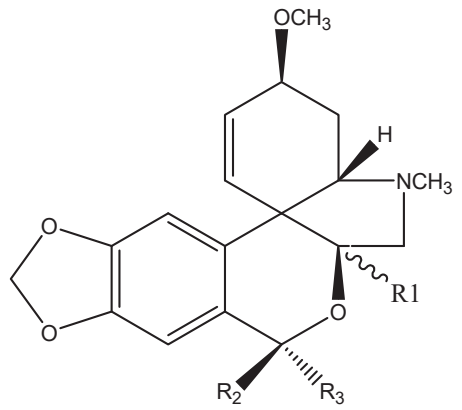

$11 \mathrm{R}_{1}=\alpha \mathrm{H} \mathrm{R}_{2}=\mathrm{R}_{3}=\mathrm{H}$<smiles></smiles>

13<smiles>[R6]Oc1ccc2c3c1O[C@H]1CC([R8])([R6])C=C[C@]31CCN([R8])C2</smiles>

$4 \mathrm{R}_{1}=\mathrm{OH}, \mathrm{R}_{2}=\mathrm{H}, \mathrm{R}_{3}=\mathrm{R}_{4}=\mathrm{CH}_{3}$

$5 \mathrm{R}_{1}=\mathrm{OH}, \mathrm{R}_{2}=\mathrm{H}, \mathrm{R}_{3}=\mathrm{CH}_{3}, \mathrm{R}_{4}=\mathrm{H}$

$6 \mathrm{R}_{1}=\mathrm{OH}, \mathrm{R}_{2}=\mathrm{R}_{3}=\mathrm{H}, \mathrm{R}_{4}=\mathrm{CH}_{3}$

$7 \mathrm{R}_{1}=\mathrm{H}, \mathrm{R}_{2}=\mathrm{OH}, \mathrm{R}_{3}=\mathrm{R}_{4}=\mathrm{CH}_{3}$

$8 \mathrm{R}_{1}+\mathrm{R}_{2}=\mathrm{O}, \mathrm{R}_{3}=\mathrm{R}_{4}=\mathrm{CH}_{3}$

$10 \mathrm{R}_{1}=\mathrm{OAc}, \mathrm{R}_{2}=\mathrm{H}, \mathrm{R}_{3}=\mathrm{R}_{4}=\mathrm{CH}_{3}$

$23 \mathrm{R}_{1}=\mathrm{OCOCH}_{2} \mathrm{CHOHCH}_{3} \mathrm{R}_{2}=\mathrm{H}, \mathrm{R}_{3}=\mathrm{R}_{4}=\mathrm{CH}_{3}$

$24 \mathrm{R}_{1}=\mathrm{OCOCH}_{2} \mathrm{CH}\left(\mathrm{OCOCH}_{3}\right) \mathrm{CH}_{3} \mathrm{R}_{2}=\mathrm{H}, \mathrm{R}_{3}=\mathrm{R}_{4}=\mathrm{CH}_{3}$<smiles>[R6]c1cc2c(cc1[R6])[C@H](P)[C@@H]([2H])C=C1CCN[C@]1(C)[C@H]2[2H]</smiles>

$15 \mathrm{R}_{1}=\mathrm{OH}, \mathrm{R}_{2}=\mathrm{OCH}_{3}, \mathrm{R}_{3}=\mathrm{R}_{4}=\mathrm{CH}_{3}$

$16 \mathrm{R}_{1}=\mathrm{OAc}, \mathrm{R}_{2}=\mathrm{OH}, \mathrm{R}_{3}=\mathrm{R}_{4}=\mathrm{CH}_{3}$

$18 \mathrm{R}_{1}=\mathrm{R}_{2}=\mathrm{OH}, \mathrm{R}_{3}+\mathrm{R}_{4}=\mathrm{CH}_{2}$

$19 \mathrm{R}_{1}=\mathrm{R}_{2}=\mathrm{OH}, \mathrm{R}_{3}=\mathrm{R}_{4}=\mathrm{CH}_{3}$

$20 \mathrm{R}_{1}=\mathrm{OAc}, \mathrm{R}_{2}=\mathrm{OH}, \mathrm{R}_{3}=\mathrm{H}, \mathrm{R}_{4}=\mathrm{CH}_{3}$

$22 \mathrm{R}_{1}=\mathrm{OH}, \mathrm{R}_{2}=\mathrm{OAc}, \mathrm{R}_{3}+\mathrm{R}_{4}=\mathrm{CH}_{2}$

$25 \mathrm{R}_{1}=\mathrm{OH}, \mathrm{R}_{2}=\mathrm{OCOC}_{3} \mathrm{H}_{5}, \mathrm{R}_{3}+\mathrm{R}_{4}=\mathrm{CH}_{2}$

$27 \mathrm{R}_{1}=\mathrm{OH}, \mathrm{R}_{2}=\mathrm{OCOC}_{3} \mathrm{H}_{6} \mathrm{OH}, \mathrm{R}_{3}+\mathrm{R}_{4}=\mathrm{CH}_{2}$

$28 \mathrm{R}_{1}=\mathrm{OH}, \mathrm{R}_{2}=\mathrm{OCOCH}_{2} \mathrm{CH}\left(\mathrm{OCOCH}_{3}\right) \mathrm{CH}_{3}, \mathrm{R}_{3}+\mathrm{R}_{4}=\mathrm{CH}_{2}$

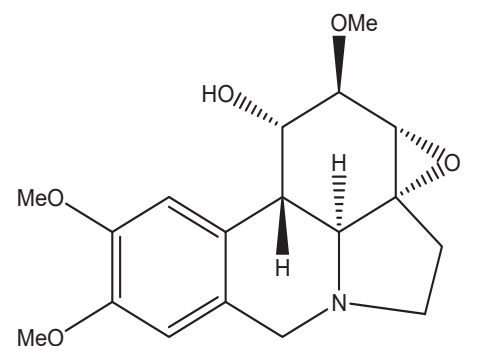

17

Figure 1. Structures of alkaloids identified in G. woronowii: Tyramine (1), hordenine (2), 5,6-dihydrobicolorine (3), galanthamine (4), sanguinine (5), $N$-demethylgalanthamine (6), epigalanthamine (7), narwedine (8), anhydrolycorine (9), O-acetylgalanthamine (10), deoxytazettine (11), assoanine (12), 11,12-didehydroanhydrolycorine (13), galanthine (15), 1-O-acetyl-9-O-methylpseudolycorine (16), incartine (17), lycorine (18), 9-O-methylpseudolycorine (19), sternbergine (20), 2-O-acetyllycorine (22), methylleucotamine (23), 3-O-(3'-acetoxybutanoyl) galanthamine (24), 2-O-2'-butenoyllycorine (25), 2-O-(3'-hydroxybutanoyl) lycorine (27), 2-O-(3'-Acetoxybutanoyl) lycorine (28) 
with diverse chemical structures $[3,4,7,9]$. This may be due to several factors including polymorphism and exogenous influences such as collection site and seasonal growth. Moreover, in support of this idea, our previous investigations on different specimens of Galanthus woronowii collected from different localities revealed that the quantities of bioactive alkaloids lycorine and galanthamine ranged between 0.008 $0.364 \%$ and $0.003-0.506 \%$ [16].

In conclusion, it is evident that the qualitative and quantitative alkaloid composition in G. woronowii specimens shows significant variation and GC-MS is proved to be a fast and relieable technique allowing identification of alkaloids in these plants.

Both of the extracts showed potent AChE activity (aerial parts: $\mathrm{IC}_{50}=0.027 \mu \mathrm{g} / \mathrm{mL}$ and bulbs: $\mathrm{IC}_{50}=0.084 \mu \mathrm{g} / \mathrm{mL}$ ) which may be due to the presence of galanthamine- or lycorine-type Amaryllidaceae alkaloids. Especially, the presence of galanthamine $\left(\mathrm{IC}_{50}=0.15 \mu \mathrm{M}\right)$ and galanthine $\left(\mathrm{IC}_{50}=7.75 \mu \mathrm{M}\right)$ in both of the extracts may well contribute to the AChE inhibitory activity of the extracts $[7,11]$.

\section{Acknowledgments}

This study was financially supported by Ege University Research Fund (Project No: 09/ECZ/021 and 09/ECZ/018) and partially supported by TÜBİTAK (104T272).

\section{References}

1. Davis AP. The Genus Galanthus. Editor: Mathew B. Timber Pres Inc., Oregon. 1999.

2. Davis AP. The Genus Galanthus-snowdrops in the wild. In: Snowdrops, A Monograph of Cultivated Galanthus. Editors: Bishop M, Davis AP, Grimshaw J. Griffin Press Publishing Ltd, Cheltenham. 2006, pp. 9-63.

3. Ünver N. New skeletons and new concepts in Amaryllidaceae alkaloids. Phytochem Rev 2007; 6: 125-35.

4. Berkov S, Codina C, Bastida J. The Genus Galanthus: A Source of Bioactive Compounds. In: Phytochemicals - A Global Perspective of Their Role in Nutrition and Health. Editor: Rao V. InTech. 2012, pp. 235-254.

5. Yakovleva A. Alkaloids of Galanthus woronovii. VI Isolation of tazettine. Zhurnal Obshchei Khimii 1963; 33: 1691-3.

6. Kintsurashvili L, Vachnadze V. Plants of the Amaryllidaceae family grown and introduced in Georgia: A source of galanthamine. Pharm Chem 2007; 41: 492-4.

7. Bozkurt-Sarıkaya B, Kaya Gİ, Önür MA, Bastida J, ÜnverSomer N. Phytochemical investigation of Galanthus woronowii. Biochem Syst Ecol 2013; 51: 276-9.

8. Ellman L, Courtney KD, Andres VJr, Featherstone RM. New and rapid colorimetric determination of acetylcholinesterase activity. Biochem. Pharmacol 1961; 7: 88-95.

9. Bozkurt-Sarıkaya B, Ünver-Somer N, Kaya Gİ, Önür MA, Bastida J, Berkov S. GC-MS investigation and acetylcholinesterase inhibitory activity Galanthus rizehensis. Z. Naturforsch 2013; 68c: 118-24.

10. López S, Bastida J, Viladomat F, Codina C. Acetylcholinesterase inhibitory activity of some Amaryllidaceae alkaloids and Narcissus extracts. Life Sci 2002; 71: 2521-9.

11. Bastida J, Lavilla R, Viladomat F. Chemical and biological aspects of Narcissus alkaloids. In: The Alkaloids chemistry and biology. Editor: Cordell GA. Elsevier Inc, San Diago. 2006, 63: pp. 87-179.

12. Çitoğlu G, Acıkara OB, Yılmaz BS, Özbek H. Evaluation of analgesic, anti-inflammatory and hepatoprotective effects of lycorine from Sternbergia fisheriana (Herbert) Rupr. Fitoterapia 2012; 83: 81-7.

13. Şenel G, Özkan M, Kandemir N. A karyological investigation on some rare and endangered species of Amaryllidaceae in Turkey. Pak J Bot 2002; 34: 229-35.

14. Zeybek N. Taxonomical investigations on Turkish snowdrops (Galanthus L.). Turk J Botany 1988;12: 89-103.

15. Zeybek N, Sauer E. Turkish Snowdrops I (Beitrag zur Kenntnis der Türkischen Schneeglöckhen)(Galanthus L.) I. Ornamental Plants. Agriculture and Industry Press, Izmir. 1994.

16. Emir A, Çiçek Polat D, Kaya Gİ, Sarıkaya B, Önür MA, Unver Somer N. Simultaneous Determination of Lycorine and Galanthamine in Galanthus woronowii by HPLC-DAD. Acta Chromatogr 2013;25: 755-64.

17. Berkov S, Bastida J, Nikolova M, Viladomat F, Codina C. Rapid TLC/GC-MS identification of acetylcholinesterase inhibitors in alkaloid extracts. Phytochemistry Anal 2008; 19: 411-9.

18. Berkov S, Bastida J, Sidjimova B, Viladomat F, Codina C. Phytochemical differentiation of Galanthus nivalis and Galanthus elwesii (Amaryllidaceae): A case study. Biochem Syst Ecol 2008; 36: 638-45.

19. Viladomat F, Bastida J, Tribo G, Codina C, Rubiralta M. Alkaloids from Narcissus bicolor. Phytochemistry 1990; 29: 1307-10.

20. Berkov S, Bastida J, Viladomat F. Codina C. Analysis of galanthamine type- alkaloids by capillary gas chromatographymass spectrometry in plants. Phytochemistry Anal 2008; 19: 285-93.

21. Berkov S, Evstatieva L, Popov S. Alkaloids in Bulgarian Pancratium maritimum L. Z. Naturforsch 2004; 59c: 65-9.

22. Berkov S, Pavlov A, Ilieva M, Burrus M, Popov S. Stanilova M. CGC-MS of alkaloids in Leucojum aestivum plants and their in vitro cultures. Phytochemistry Anal 2005; 16: 98-103.

23. Kreh M, Matusch R, Witte L. Acetylated alkaloids from Narcissus pseudonarcissus. Phytochemistry 1995; 40: 1303-6.

24. Berkov S, Bastida J, Tsvetkova R, Viladomat F. Alkaloids from Sternbergia colchiciflora. Z. Naturforsch 2009; 64c: 311-6.

25. Evidente A, Iasiello I, Randazzo G. Isolation of sternbergine, a new alkaloid from bulbs of Sternbergia lutea. J Nat Prod 1984; 47: 1003-8.

26. Cedron JC, Gutiérrez D, Flores N, Ravelo AG, EstevezBraun A. Synthesis and antiplasmodial activity of lycorine derivatives. Bioorg Med Chem 2010; 18: 4694-4701. 
27. Toriizuka Y, Kinoshita E, Kogure N, Kitajima M, Ishiyama A, Otoguro K, Yamada H, Omura S, Takayama H. New lycorine-type alkaloid from Lycoris traubii and evaluation of antitrypanosomal and antimalarial acitivities of lycorine derivatives. Bioorg Med Chem 2008; 16: 10182-9.

28. Berkov S, Viladomat F, Codina C, Suarez S, Ravelo A, Bastida J. GC-MS of Amaryllidaceous galanthamine-type alkaloids. J Mass Spectrom 2012; 47: 1065-73.
29. Berkov S, Cuadrado M, Osorio E, Viladomat F, Codina C, Bastida J. Three new alkaloids from Galanthus nivalis and Galanthus elwesii. Planta Med 2009; 75: 1351-5.

30. Torras-Claveria L, Berkov S, Codina C, Viladomat F, Bastida J. Metabolomic analysis of bioactive Amaryllidaceae alkaloids of ornamental varieties of Narcissus by GC-MS combined with k-means cluster analysis. Ind Crop Prod 2014; 56: 211-22. 